\title{
REVIEW
}

\section{NURSING WORK ENVIRONMENT AND UNFINISHED NURSING CARE IN HOSPITAL SETTINGS - A SCOPING REVIEW}

\author{
Elena Gurková $(D$, Daniela Bartoníčková, Zdeňka Mikšová \\ Department of Nursing, Faculty of Health Sciences, Palacký University in Olomouc, Czech Republic
}

Received July 14, 2020; Accepted March 3, 2021. Copyright: This is an open access article under the CC BY-NC-ND license.

\begin{abstract}
Aim: This scoping review aimed to summarize the evidence regarding the relationship between the nursing work environment and unfinished nursing care, and to offer a deeper insight into organizational factors of unfinished nursing care in hospital settings. A further aim of the study was to collate evidence about instruments for measurement of nurses' perceptions of the nursing work environment. Design: A scoping review was performed. Methods: A search was conducted in eight scientific databases. The final review included 22 studies and publications ranging from 2010 to 2020 . Guidelines regarding scoping review methodology developed by the Joanna Briggs Institute were followed in our study. Results: The majority of studies included representative hospital and nurse samples, and had a cross-sectional design. Evidence indicates that the nursing work environment is a significant predictor of unfinished nursing care. The more favorable the nursing work environment, as perceived by nurses, the less frequently they reported unfinished nursing care events. The domains of the Practice Environment Scale of the Nursing Work Index were found to be significant predictors of unfinished nursing care. Conclusion: Nurses working in favorable conditions reported lower prevalence of unfinished nursing care. Improvements in staffing and resource adequacy were found to be the most significant factors predicting lower rates of unfinished nursing care. Refinement of factors in the nursing work environment and staffing are interventions that can mitigate unfinished nursing care. Further research should focus on the relationship between different dimensions of the nursing work environment and unfinished nursing care in hospital settings.
\end{abstract}

Keywords: implicit rationing of nursing care, missed nursing care, nurse work environment, scoping review, unfinished nursing care.

\section{Introduction}

Unfinished nursing care (UNC), (or other interchangeably used terms such as "implicit rationing", "missed nursing care", and "care left undone") is a global phenomenon in health care, negatively associated with patient safety culture, quality of nursing care and nurse-related outcomes, such as nurse retention and job satisfaction (Jones et al., 2019). The predicted worldwide nursing shortage emphasizes the urgent need to facilitate a better understanding of this phenomenon in the international professional nursing community (Gustafsson et al., 2020). Indeed, there has been a growing body of research, over the last two decades, investigating the multifactorial nature of the phenomenon of UNC and cause-effect relationships with UNC, since they determine the type of interventions that can mitigate UNC events. Antecedents or contributing factors of UNC are defined and understood through influential; conceptual frameworks of UNC (Bail

Corresponding author: Elena Gurková, Department of Nursing, Faculty of Health Sciences, Palacký University in Olomouc, Hněvotínská 976/3, Olomouc, Czech Republic; email: elena.gurkova@upol.cz.
\& Grealish, 2016; Jones et al., 2015, 2019; Kalisch et al., 2009; Lucero et al., 2009; Schubert et al., 2007), which have been investigated across a variety of clinical settings, health care systems and cultures. In conceptual frameworks of UNC (Bail \& Grealish, 2016; Kalish et al., 2009; Schubert et al., 2007), and in some measurements of this concept (e.g. the MISSCARE Survey, Kalisch \& Williams, 2009), important variables related to the practice or the nursing work environment (NWE) have been established, including staffing and skill mix, interdisciplinary collaboration, autonomy and responsibility, nursing management, institutional organization and support, sufficiency of resources, and communication and teamwork (Zhao et al., 2020).

Rigorous hypothetical models explaining the multifactorial nature of this phenomenon have been replicated and tested in international studies, and several reasons why nursing care is neglected or delayed have been reported (Ausserhofer et al., 2014; Blackman et al., 2015, 2018). Contributing factors to, or predictors of UNC are important areas of empirical studies across different countries and multiple practice settings (Campbell et al., 2020; Lake 
et al., 2020a; Jones et al., 2019). Predictors of, or contributing factors to UNC represent a complex mixture of nurse and patient characteristics, hospital and unit characteristics, organizational variables and attributes of NWE, and care philosophy that can negatively affect nurses' capacity to carry out care activities (Jones et al., 2015). Therefore, the research emphasis has predominantly focused on nursing human resources, various organisational variables (the nurse-patient ratio, perception of staffing or resources, type of the unit or hospital, and different shifts) and other aspects of the NWE (Ausserhofer et al., 2014; Blackman et al., 2015, 2018; Bragadóttir et al., 2017; Campbell et al., 2020; Duffy et al., 2018; Kalisch \& Lee, 2012; Lake et al., 2020a, 2020b; Liu et al., 2018; Park et al., 2018; Zhao et al., 2020).

NWE characteristics were confirmed in recent research syntheses (Griffiths et al., 2020; Jones et al., 2015) as a stronger predictor of UNC than individual nurse variables (such as gender, age, education, experience, work role etc.). Current research syntheses (Cordeiro et al., 2020; Griffiths et al., 2020; Jones et al., 2015; Papastavrou et al., 2014a; Zhao et al., 2020) have identified two common organizational determinants of UNC - the NWE and staffing. Moreover, these reviews have discussed how supportive work environments with increased teamwork and effective communication between all health professionals, and adequate staffing are linked to lower UNC (Cordeiro et al., 2020; Zhao et al., 2020; Lake et al., 2020b). However, Zhao et al. (2020) have argued that previous empirical studies and syntheses failed to systematically explore the association between specific domains of the nursing work environment and UNC. This scoping review contributes to the existing evidence with a deeper insight into what is known about the association between specific domains of the NWE and UNC in hospital settings, and how nurses' perceptions of the NWE have been assessed in hospital settings. The purpose of the analysis is to deepen our understanding of the relationship between these two constructs, which could provide a basis for the type of interventions and implementation of supportive strategies for mitigating UNC and adverse events.

\footnotetext{
Aim

This scoping review adds to the existing literature by examining how the NWE has been investigated, and what domains of the NWE have an impact on UNC. The analysis aims to summarize the evidence regarding the relationship between the NWE and $\mathrm{UNC}$, and to offer a deeper insight into organizational factors of UNC in a hospital context.
}

\section{Methods}

\section{Design}

Guidelines regarding scoping review methodology developed by the Joanna Briggs Institute (Peters et al., 2015), and the PRISMA-Sco checklist (Tricco et al., 2016) for scoping review were followed in our study. Five methodological steps for scoping reviews were applied: identifying a research question; identifying relevant studies; selecting studies; collecting data; summarizing data, and synthesizing results.

The following questions were specified to guide the scoping review:

1. What is known about the association between the NWE and UNC in hospital settings?

2. How have nurses' perceptions of the NWE been assessed in hospital settings?

3. Which instruments have been used to measure nurses' perceptions of the NWE in hospital settings?

\section{Eligibility criteria}

Selection of relevant studies began with specification of the inclusion criteria. After the initial search was carried out, qualitative and quantitative approaches in studies were identified. The qualitative approach examines how nurses working in acute-care hospitals experience UNC. Qualitative studies focus mainly on nurses' decision-making processes within increasing time constraints (e.g. Harvey et al., 2020). The qualitative studies did not directly relate to the research questions and were excluded from the analysis.

Studies were eligible for inclusion if they: a) reported the measurement of variables related to the NWE and UNC in hospital settings; b) included quantitative empirical results about the association between UNC and the NWE in hospital settings; c) were peerreviewed and the full text was available; d) featured nursing staff in hospitals as participants. Studies without specific instruments measuring nurses' perception of the NWE or UNC, discussion papers, reviews, editorials, conference abstracts, books, reports, and dissertations were excluded.

\section{Search strategy}

A search of the literature was conducted in June 2020. The electronic databases Web of Science, PROQUEST, EBSCOhost Research Databases Platform, EBM Reviews, MEDLINE Complete, ScienceDirect, Scopus, and PsycInfo were used to gather data for a review of quantitative studies.

The search was limited to publications in English at title / abstract level, and only studies published 
between 2000 (when the first influential nursing studies focusing on organizational attributes of hospitals were published) and the first quarter of 2020 were included. In the initial stage of the scoping review, a search in EBSCOhost Research Databases Platform was carried out to identify the search terms that would be most pertinent to the research questions. In the second step, the following search terms were used to cover all concepts emerging from contemporary nursing research regarding the umbrella term UNC: ("implicit rationing of nursing care" OR "missed nursing care" OR "unfinished nursing care" OR "nursing care left undone" OR "bedside rationing" OR "unmet nursing care needs" OR "task incompletion" OR "failure to maintain" OR "omitted care") AND ("working environment" OR "practice environment" OR "organizational variables" OR "organizational climate" OR "nurse work environment"). In addition to the database search, a manual search of the references of the obtained articles, and a scan through the Google Scholar database were conducted. A further search through grey literature, or sources in other languages were not considered. In total, the search produced 2,789 sources.

\section{Study selection inc. PRISMA flow diagram}

A total of 2,789 studies were retrieved, from which 722 duplicates were removed. Studies were first screened by title and subsequently by abstract, leading to the exclusion of 2,023 articles. A total of 44 papers were read in full text. Only 22 studies were included in the final analysis. The retrieval process is depicted in Figure 1.

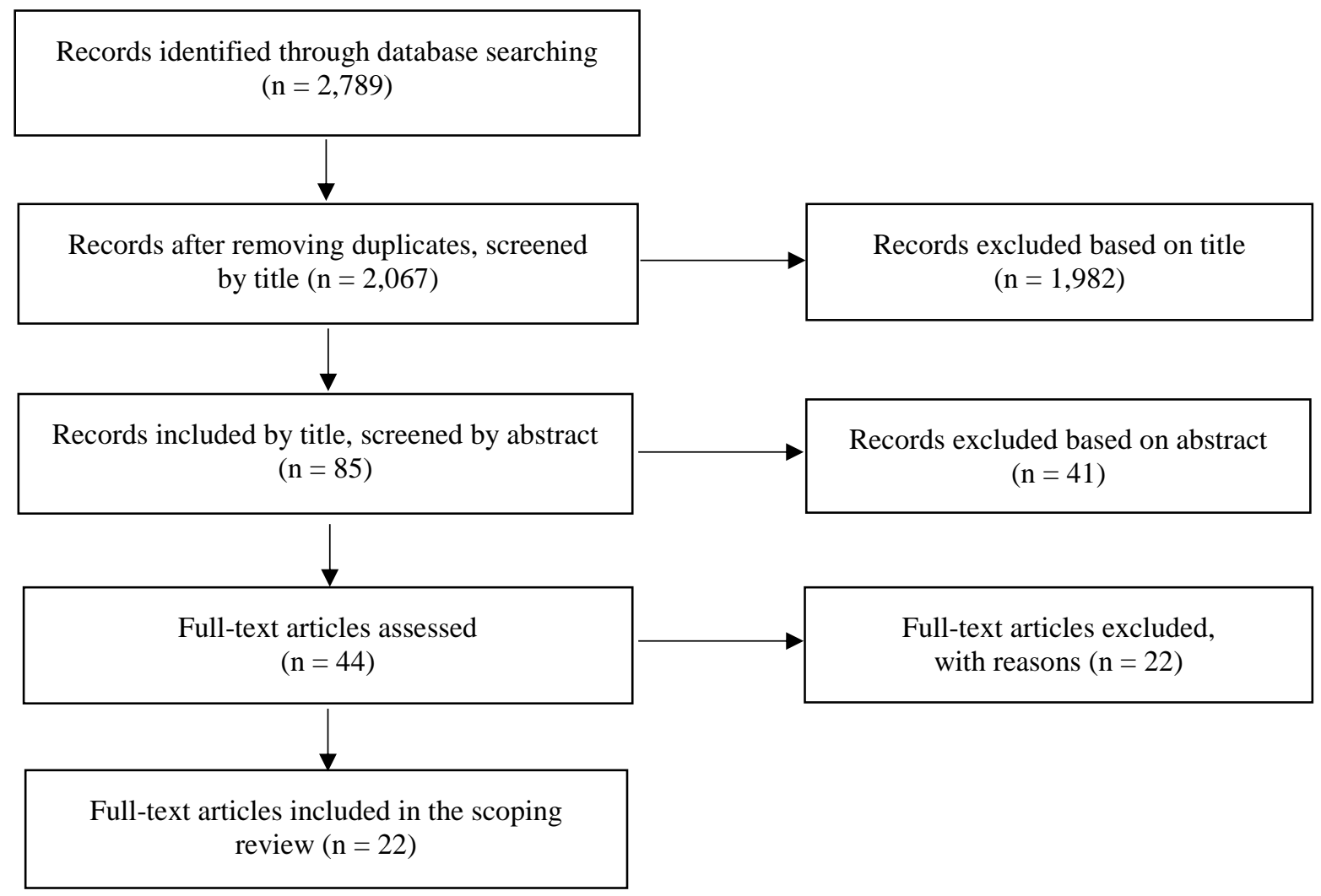

Figure 1 Flow diagram of the retrieval process

A substantial number of quantitative studies reported UNC and the NWE associated with patient or nurse outcomes. These studies did not clearly match the research questions (the relationship between specific domains of the NWE and UNC, and measurement of the NWE). Therefore, studies reporting the relationship between UNC and hospital safety climate or between UNC, the NWE and patient-centred care or patient and nurse outcomes were excluded. In addition, studies focusing solely on the relationship between UNC, staffing adequacy, or workload which did not report associations with the work environment were excluded. Studies conducted in nursing homes or using only instruments for measuring UNC without tools for measuring the NWE were also excluded. Instruments for measurement of nurses' perceptions of the NWE in hospital settings were only those extracted from the included studies. 


\section{Evaluation of quality of articles}

The results of studies were retrieved and synthesized by two independent reviewers.

Data were abstracted on sample characteristics (participants, type of hospital and unit), NWE and UNC measurements, methods of data analysis, main results, validity / reliability). These variables were cardinal to the research questions.

\section{Data extraction}

Data were further analysed by using a descriptive numerical and qualitative content analysis, which was organised and reported thematically with the stated research questions (Gustafsson et al., 2020).

\section{Results}

\section{Characteristics of included studies}

The final review included 22 studies and publications ranging from 2010 to 2020 . Ten of the included studies were conducted in the United States, one in Canada, in Brazil, in Australia, and in South Korea, and eight in European countries (Cyprus, Czech Republic, Iceland, Switzerland, United Kingdom, and Sweden). One study was an influential multinational study - the RN4CAST (Nurse forecasting: human resources planning in nursing) study (Ausserhofer et al., 2014), conducted in 12 European countries. Four European studies (one in Switzerland, two in the United Kingdom, and one in Sweden) were published as part of the RN4CAST study (Table 1). The majority of studies used regression models (linear or logistic) for data analysis.

The sample size in the studies ranged from 29 to 33,659 professional nurses. The number of participating hospitals ranged from one to 488 (Ausserhofer et al., 2014). Some studies conducted in the United States employed data from the MultiState Nursing Care and Patient Safety Survey (Brooks Carthon et al., 2015; Lake et al., 2017; 2020a, 2020b), or used secondary data from the National Database of Nursing Quality Indicators ${ }^{\circledR}$ (e.g.: Park et al., 2018; Smith et al., 2020a). Most studies used specific settings only, such as medical-surgical units, intensive care units (ICUs), neonatal or pediatric ICUs (NICUs / PICUs), general pediatric setting, and labor and delivery units (Table 1). Twenty-one studies had a cross-sectional design, and the remaining study was a two-period panel study in which participants were examined ten years apart (Lake at al., 2020b). Therefore, the evidence base was limited to crosssectional descriptive studies that did not permit causal inference.

The majority of studies included representative hospital and nurse samples (mainly studies which were conducted according to the RN4CAST protocol - Aiken et al., 2018; Ausserhofer et al., 2014; Ball et al., 2014, 2016; Schubert et al., 2013).

Characteristics of instruments used to measure nurses' perception of the NWE and UNC

Five studies (published as substudies of the RN4CAST study) used the conceptual framework developed by Schubert et al. (2007). Four of these substudies used the Tasks Undone survey with thirteen items (TU-13) for measurement of UNC. However, this framework, based on the implicit rationing approach, is represented by another parent instrument - the Basal Extent of Rationing of Nursing Care (BERNCA) Instrument (Schubert et al., 2007). The list of thirteen items in the TU-13 was informed by the BERNCA (Schubert et al., 2007). The task undone approach, using task undone tools, was applied in eight studies (Brooks Carthon et al., 2015; Hessels et al., 2015; Lake et al., 2017, 2018, 2020a; Park et al., 2018; Smith et al., 2020a).

Six studies (Bragadóttir et al., 2017; Duffy et al., 2018; Kim et al., 2018; Pereira et al., 2020, Smith et al., 2020b; Zeleníková et al., 2020) used the missed care approach. This approach is represented mainly by the MISSCARE Survey (Kalisch \& Williams, 2009). Three studies (Campbell et al., 2020; Papastavrou et al., 2014b; Schubert et al., 2013) used implicit rationing of nursing care, represented by the BERNCA or the Perceived Implicit Rationing of Nursing Care survey (PIRNCA, Jones, 2014). Three studies were conducted on NICUs and PICUs using specific tools (the Neonatal Extent of Work Rationing Instrument NEWRI or TU with 17 items) (Rochefort \& Clarke, 2010; Smith et al., 2020a), or using the MISSCARE instrument (Pereira et al., 2020). The most frequently used instrument for the NWE was the Practice Environment Scale of the Nursing Work Index (PESNWI), applied in 17 studies. One study used the Nursing Teamwork Survey (Bragadóttir et al., 2017), two studies used the Revised Nursing Work Index (NWI-R, Rochefort \& Clarke, 2010; Smith et al., 2020a), and in the remaining two studies, the NWE was measured by the Revised Professional Practice Environment (RPPE, Papastavrou et al., 2014b; Zeleníková et al., 2020).

\section{Relationship between the NWE and UNC}

All included studies confirmed unambiguously that a favorable NWE had a significant inverse relationship with UNC. Only two studies showed a low association between UNC and the NWE (Papastavrou et al., 2014b; Zeleníková et al., 2020). However, the RPPE was used in these two studies for assessing domains of the NWE. 
Results of studies in which the PES-NWI was used have shown significant associations between these two constructs. However, most studies calculated a composite score averaging scores of the dimensions to delineate the overall NWE. Seven studies (Campbell et al., 2020; Kim et al., 2018; Park et al.,
2018; Rochefort \& Clarke, 2010; Schubert et al., 2013; Smith et al., 2020a; Zeleníková et al., 2020) evaluated associations between domains of the PES-NWI and UNC. Improved staffing and resource adequacy were found to be the most significant factors predicting lower prevalence of UNC.

Table 1 Description of studies used in the scoping review (Part 1)

\begin{tabular}{|c|c|c|c|c|c|c|}
\hline Study & $\begin{array}{l}\mathrm{NWE} \\
\text { measurements }\end{array}$ & $\begin{array}{l}\text { UNC } \\
\text { measurements }\end{array}$ & $\begin{array}{l}\text { Participants, } \\
\text { hospitals and } \\
\text { units }\end{array}$ & Data analysis & Main results & $\begin{array}{l}\text { Validity / } \\
\text { reliability }\end{array}$ \\
\hline $\begin{array}{l}\text { Aiken et al. } \\
\text { (2018), UK } \\
\text { RN4CAST- } \\
\text { England study }\end{array}$ & PES-NWI & $\begin{array}{l}\text { RN4CAST } \\
\text { Survey } \\
\text { UNC was } \\
\text { measured by } \\
\text { the TU tool } \\
\text { with } 13 \text { items - } \\
\text { TU } 13\end{array}$ & $\begin{array}{l}\text { A representative } \\
\text { sample of 2,963 } \\
\text { RNs from } 31 \\
\text { NHS trusts and } 46 \\
\text { hospitals was } \\
\text { used. } \\
\text { General medical, } \\
\text { surgical or mixed } \\
\text { medical-surgical } \\
\text { units were } \\
\text { randomly } \\
\text { selected. }\end{array}$ & $\begin{array}{l}\text { Nurse survey } \\
\text { data were used } \\
\text { from the } 2010 \\
\text { RN4CAST- } \\
\text { England study. } \\
\text { Regression } \\
\text { models with and } \\
\text { without control } \\
\text { variables were } \\
\text { performed. }\end{array}$ & $\begin{array}{l}\text { Significant } \\
\text { association was } \\
\text { found between } \\
\text { UNC and } \\
\text { NWE. } \\
\text { Better work } \\
\text { environments } \\
\text { were related to } \\
\text { lower UNC } \\
\text { (b=-0.26, } \\
95 \% \text {; } \\
\text { CI: }-0.48 \text { to } \\
-0.04) \text {. }\end{array}$ & $\begin{array}{l}\text { The cross- } \\
\text { sectional } \\
\text { design allows } \\
\text { for no causal } \\
\text { inferences. } \\
\text { Validated } \\
\text { measurements } \\
\text { were used. }\end{array}$ \\
\hline $\begin{array}{l}\text { Ausserhofer } \\
\text { et al. (2014) } \\
\text { RN4CAST- } \\
\text { study } \\
12 \text { European } \\
\text { countries } \\
\text { (Belgium, } \\
\text { England, } \\
\text { Finland, } \\
\text { Germany, } \\
\text { Greece, } \\
\text { Ireland, The } \\
\text { Netherlands, } \\
\text { Norway, } \\
\text { Poland, Spain, } \\
\text { Sweden and } \\
\text { Switzerland) }\end{array}$ & PES-NWI & $\begin{array}{l}\text { RN4CAST } \\
\text { Survey } \\
\text { TU } 13\end{array}$ & $\begin{array}{l}\text { A representative } \\
\text { sample of } 33,659 \\
\text { professional } \\
\text { nurses from } 12 \\
\text { countries and } 488 \\
\text { hospitals was } \\
\text { used. } \\
\text { General medical, } \\
\text { surgical or mixed } \\
\text { medical-surgical } \\
\text { units were } \\
\text { randomly } \\
\text { selected. }\end{array}$ & $\begin{array}{l}\text { Nurse survey } \\
\text { data were used } \\
\text { from the } \\
\text { RN4CAST- } \\
\text { study } \\
\text { (2009-2011). } \\
\text { A multiple } \\
\text { multilevel linear } \\
\text { regression model } \\
\text { was employed. } \\
\text { Simple three- } \\
\text { level regression } \\
\text { models were } \\
\text { applied to test } \\
\text { the associations } \\
\text { between the } \\
\text { covariates } \\
\text { (NWE) and } \\
\text { UNC, and then } \\
\text { multiple three- } \\
\text { level regression } \\
\text { analyses were } \\
\text { performed. }\end{array}$ & $\begin{array}{l}\text { Significant } \\
\text { association } \\
\text { were found } \\
\text { between UNC } \\
\text { and NWE. } \\
\text { Better work } \\
\text { environments } \\
\text { were related to } \\
\text { lower UNC } \\
(b=-2.19 ; \\
p<0.0001) .\end{array}$ & $\begin{array}{l}\text { The cross- } \\
\text { sectional } \\
\text { design allows } \\
\text { for no causal } \\
\text { inferences. } \\
\text { Validated } \\
\text { measurements } \\
\text { were used. } \\
\text { Random } \\
\text { effects were } \\
\text { included for } \\
\text { the hospital } \\
\text { and country } \\
\text { level. }\end{array}$ \\
\hline $\begin{array}{l}\text { Ball et al. } \\
\text { (2014), UK }\end{array}$ & PES-NWI & $\begin{array}{l}\text { RN4CAST } \\
\text { Survey } \\
\text { TU } 13\end{array}$ & $\begin{array}{l}\text { A representative } \\
\text { sample of } 2,917 \\
\text { RNs and } \\
\text { unregistered nurse } \\
\text { support workers } \\
\text { or HCSW from } \\
46 \text { hospitals ( } 401 \\
\text { wards) was used. } \\
\text { Mixed medical- } \\
\text { surgical units } \\
\text { were selected. }\end{array}$ & $\begin{array}{l}\text { Nurse survey } \\
\text { data were } \\
\text { collected from } \\
\text { RNs in England } \\
\text { according to the } \\
\text { RN4CAST } \\
\text { protocol. Multi- } \\
\text { level regression } \\
\text { models were } \\
\text { performed. }\end{array}$ & $\begin{array}{l}\text { Negative } \\
\text { associations } \\
\text { were found } \\
\text { between UNC } \\
\text { and NWE. } \\
\text { The more } \\
\text { favourable } \\
\text { NWE perceived } \\
\text { by nurses, the } \\
\text { less frequently } \\
\text { they reported } \\
\text { UNC events } \\
(r=-0.32 ; \\
p<0.001) .\end{array}$ & $\begin{array}{l}\text { The cross- } \\
\text { sectional } \\
\text { design allows } \\
\text { for no causal } \\
\text { inferences. } \\
\text { Validated } \\
\text { measurements } \\
\text { were used. }\end{array}$ \\
\hline
\end{tabular}


Table 2 Description of studies used in the scoping review (Part 2)

\begin{tabular}{|c|c|c|c|c|c|c|}
\hline Study & $\begin{array}{l}\mathrm{NWE} \\
\text { measurements }\end{array}$ & $\begin{array}{l}\text { UNC } \\
\text { measurements }\end{array}$ & $\begin{array}{l}\text { Participants, } \\
\text { hospitals and } \\
\text { units }\end{array}$ & Data analysis & Main results & $\begin{array}{l}\text { Validity / } \\
\text { reliability }\end{array}$ \\
\hline $\begin{array}{l}\text { Ball et al. } \\
(2016) \text {, } \\
\text { Sweden }\end{array}$ & PES-NWI & $\begin{array}{l}\text { RN4CAST } \\
\text { Survey } \\
\text { TU } 13\end{array}$ & $\begin{array}{l}\text { A representative } \\
\text { sample of } 10,174 \\
\text { RNs and } \\
\text { unregistered nurse } \\
\text { support workers } \\
\text { from } 79 \text { acute } \\
\text { hospitals was used. } \\
\text { General medical, } \\
\text { surgical units were } \\
\text { selected. }\end{array}$ & $\begin{array}{l}\text { Nurse survey } \\
\text { data were from } \\
\text { the RN4CAST- } \\
\text { study } \\
\text { conducted in } \\
\text { Sweden } \\
(2010) \text {. Two } \\
\text { multi-level } \\
\text { regression } \\
\text { models were } \\
\text { performed. }\end{array}$ & $\begin{array}{l}\text { Negative } \\
\text { associations were } \\
\text { found between } \\
\text { UNC and NEW } \\
(\mathrm{OR}=-0.516 \text {; } \\
\mathrm{p}<0.001)\end{array}$ & $\begin{array}{l}\text { The cross- } \\
\text { sectional design } \\
\text { allows for no } \\
\text { causal } \\
\text { inferences. } \\
\text { Validated } \\
\text { measurements } \\
\text { were used. }\end{array}$ \\
\hline $\begin{array}{l}\text { Bragadóttir } \\
\text { et al. } \\
\text { (2017), } \\
\text { Iceland }\end{array}$ & $\begin{array}{l}\text { Nursing } \\
\text { Teamwork } \\
\text { Survey- } \\
\text { Icelandic }\end{array}$ & $\begin{array}{l}\text { The } \\
\text { MISSCARE } \\
\text { Survey }\end{array}$ & $\begin{array}{l}\text { A representative } \\
\text { sample of } 867 \text { RNs } \\
\text { from } 27 \text { medical, } \\
\text { surgical and ICU } \\
\text { inpatient units in } 8 \\
\text { hospitals in Iceland } \\
\text { was obtained. }\end{array}$ & $\begin{array}{l}\text { Correlational } \\
\text { and regression } \\
\text { statistics were } \\
\text { employed. }\end{array}$ & $\begin{array}{l}\text { Nursing teamwork } \\
\text { was found to be } \\
\text { a significant } \\
\text { predictor of UNC } \\
\text { (predicted } 14 \% \text { of } \\
\text { the variance in } \\
\text { UNC after } \\
\text { controlling for unit } \\
\text { type, role, age and } \\
\text { staffing } \\
\text { adequacy). }\end{array}$ & $\begin{array}{l}\text { The cross- } \\
\text { sectional design } \\
\text { allows for no } \\
\text { causal } \\
\text { inferences. } \\
\text { Validated } \\
\text { measurements } \\
\text { were used. }\end{array}$ \\
\hline $\begin{array}{l}\text { Campbell } \\
\text { et al. } \\
\text { (2020), } \\
\text { USA }\end{array}$ & PES-NWI & $\begin{array}{l}\text { PIRNCA } \\
\text { Survey }\end{array}$ & $\begin{array}{l}\text { A representative } \\
\text { sample of } 950 \mathrm{RNs} \\
\text { was used. } \\
\text { Medical-surgical, } \\
\text { ICU and others } \\
\text { (specialities not } \\
\text { speciated) units } \\
\text { were selected. }\end{array}$ & $\begin{array}{l}\text { Surveys were } \\
\text { administered as } \\
\text { part of the } \\
\text { Alabama } \\
\text { Hospital Staff } \\
\text { Nurse Study. } \\
\text { Multiple linear } \\
\text { regressions } \\
\text { were used. }\end{array}$ & $\begin{array}{l}\text { Negative } \\
\text { associations were } \\
\text { found between } \\
\text { UNC, five } \\
\text { subscales and a } \\
\text { composite score of } \\
\text { the PES-NWI. } \\
\text { Domains of the } \\
\text { PES-NWI were } \\
\text { found to be } \\
\text { significant } \\
\text { contributing } \\
\text { factors to UNC. }\end{array}$ & $\begin{array}{l}\text { The cross- } \\
\text { sectional design } \\
\text { allows for no } \\
\text { causal } \\
\text { inferences. } \\
\text { Validated } \\
\text { measurements } \\
\text { were used. } \\
\text { The results } \\
\text { have limited } \\
\text { generalizability } \\
\text { (data were } \\
\text { obtained only } \\
\text { from Alabama) } \\
\text { and study had a } \\
\text { low response } \\
\text { rate. PES-NWI } \\
\text { scores were } \\
\text { obtained only at } \\
\text { the individual } \\
\text { level and } \\
\text { exploration of } \\
\text { unit or hospital } \\
\text { level work } \\
\text { environments } \\
\text { was not } \\
\text { possible. }\end{array}$ \\
\hline
\end{tabular}


Table 3 Description of studies used in the scoping review (Part 3)

\begin{tabular}{|c|c|c|c|c|c|c|}
\hline Study & $\begin{array}{l}\mathrm{NWE} \\
\text { measurements }\end{array}$ & $\begin{array}{l}\text { UNC } \\
\text { measurements }\end{array}$ & $\begin{array}{l}\text { Participants, } \\
\text { hospitals and } \\
\text { units }\end{array}$ & Data analysis & Main results & $\begin{array}{l}\text { Validity / } \\
\text { reliability }\end{array}$ \\
\hline $\begin{array}{l}\text { Brooks } \\
\text { Carthon } \\
\text { et al. } \\
(2015) \text {, } \\
\text { USA }\end{array}$ & PES-NWI & $\begin{array}{l}\text { An inventory of } \\
10 \text { common } \\
\text { nursing } \\
\text { activities that } \\
\text { nurses left } \\
\text { incomplete. }\end{array}$ & $\begin{array}{l}\text { Large random } \\
\text { samples of } 20,605 \\
\text { professional bedside } \\
\text { nurses were } \\
\text { obtained from } 419 \\
\text { hospitals. The } \\
\text { purpose of the study } \\
\text { was to examine the } \\
\text { relationship between } \\
\text { the NWE, UNC and } \\
\text { heart failure } \\
\text { readmissions. The } \\
\text { type of unit was not } \\
\text { mentioned in the } \\
\text { study. }\end{array}$ & $\begin{array}{l}\text { Secondary data } \\
\text { of } 419 \text { general } \\
\text { acute care } \\
\text { hospitals in the } \\
\text { USA were } \\
\text { used. Data } \\
\text { were collected } \\
\text { through the } \\
\text { Multi-State } \\
\text { Nursing Care } \\
\text { and Patient } \\
\text { Safety Survey. } \\
\text { A set of mixed- } \\
\text { level, robust } \\
\text { fixed effects } \\
\text { logistic } \\
\text { regression } \\
\text { models were } \\
\text { performed. }\end{array}$ & $\begin{array}{l}\text { Nurses working in } \\
\text { favourable } \\
\text { conditions reported } \\
\text { lower prevalence } \\
\text { of UNC. Higher } \\
\text { percentage of } \\
\text { nurses reported } \\
\text { being unable to } \\
\text { talk to }(47.5 \% \text { vs } \\
37.1 \% ; \mathrm{p}<0.001) \text {, } \\
\text { complete care } \\
\text { plans for their } \\
\text { patients }(40.5 \% \text { vs } \\
31.9 \% \text {; }<0.001) \\
\text { or teach }(38.1 \% \text { vs } \\
25.2 \% \text {; }<0.001) \\
\text { in poor as } \\
\text { compared to good } \\
\text { working } \\
\text { environments. }\end{array}$ & $\begin{array}{l}\text { Hospital-level } \\
\text { measurements of } \\
\text { UNC were } \\
\text { calculated. } \\
\text { Validated } \\
\text { measurements } \\
\text { were used only } \\
\text { for the } \\
\text { measurement of } \\
\text { the NWE. } \\
\text { The cross- } \\
\text { sectional design } \\
\text { allows for no } \\
\text { causal } \\
\text { inferences. }\end{array}$ \\
\hline $\begin{array}{l}\text { Duffy et } \\
\text { al. } \\
\text { (2018), } \\
\text { USA }\end{array}$ & PES-NWI & $\begin{array}{l}\text { The } \\
\text { MISSCARE } \\
\text { Survey }\end{array}$ & $\begin{array}{l}\text { Small random } \\
\text { sample of RNs } \\
(\mathrm{n}=138) \text { was } \\
\text { obtained from one } \\
\text { regional referral } \\
\text { hospital. The RNs } \\
\text { were obtained from } \\
17 \text { inpatient units } \\
\text { and the emergency } \\
\text { department. }\end{array}$ & $\begin{array}{l}\text { Both } \\
\text { correlational } \\
\text { analyses and } \\
\text { stepwise } \\
\text { regression } \\
\text { analyses were } \\
\text { employed. }\end{array}$ & $\begin{array}{l}\text { Negative strong } \\
\text { associations were } \\
\text { found between } \\
\text { UNC and NEW } \\
(r=-0.477)\end{array}$ & $\begin{array}{l}\text { The cross- } \\
\text { sectional design } \\
\text { allows for no } \\
\text { causal } \\
\text { inferences. } \\
\text { Validated } \\
\text { measurements } \\
\text { were used. } \\
\text { The results have } \\
\text { limited } \\
\text { generalizability. }\end{array}$ \\
\hline $\begin{array}{l}\text { Hessels } \\
\text { et al. } \\
\text { (2015), } \\
\text { USA }\end{array}$ & PES-NWI & $\begin{array}{l}\text { RN4CAST } \\
\text { Survey } \\
\text { UNC was } \\
\text { measured by the } \\
\text { TU tool with } 12 \\
\text { items - TU } 12\end{array}$ & $\begin{array}{l}\text { A representative } \\
\text { random sample of } \\
7,679 \text { nurses from } \\
70 \text { hospitals in New } \\
\text { Jersey was used. } \\
\text { Nurses provided } \\
\text { inpatient care at the } \\
\text { bedside (the type of } \\
\text { units were not } \\
\text { mentioned). }\end{array}$ & $\begin{array}{l}\text { Authors } \\
\text { performed } \\
\text { multiple } \\
\text { regression } \\
\text { techniques with } \\
\text { robust } \\
\text { procedures } \\
\text { using Huber- } \\
\text { White } \\
\text { sandwich } \\
\text { variance } \\
\text { estimators. }\end{array}$ & $\begin{array}{l}\text { Each of the five } \\
\text { subscales and a } \\
\text { composite score of } \\
\text { the PES-NWI was } \\
\text { confirmed to be } \\
\text { the inverse } \\
\text { predictors of UNC } \\
(\beta=-0.47 \text { to }-0.77 \text {, } \\
\text { p }<0.01) \text {. For } \\
\text { every full point } \\
\text { increase in the } \\
\text { hospital score on } \\
\text { the composite } \\
\text { PES-NWI, } \\
\text { indicating a better } \\
\text { work environment, } \\
\text { there was a } 13.7 \% \\
\text { decrease in the } \\
\text { percentage of } \\
\text { necessary care that } \\
\text { is left undone by } \\
\text { nurses in hospitals. }\end{array}$ & $\begin{array}{l}\text { The cross- } \\
\text { sectional design } \\
\text { allows for no } \\
\text { causal } \\
\text { inferences. } \\
\text { Validated } \\
\text { measurements } \\
\text { were used. }\end{array}$ \\
\hline
\end{tabular}


Table 4 Description of studies used in the scoping review (Part 4)

\begin{tabular}{|c|c|c|c|c|c|c|}
\hline Study & $\begin{array}{l}\text { NWE } \\
\text { measurements }\end{array}$ & $\begin{array}{l}\text { UNC } \\
\text { measurements }\end{array}$ & $\begin{array}{l}\text { Participants, } \\
\text { hospitals and } \\
\text { units }\end{array}$ & $\begin{array}{l}\text { Data } \\
\text { analysis }\end{array}$ & Main results & $\begin{array}{l}\text { Validity / } \\
\text { reliability }\end{array}$ \\
\hline $\begin{array}{l}\text { Kim et al. } \\
(2018), \\
\text { South Korea }\end{array}$ & PES-NWI & $\begin{array}{l}\text { The } \\
\text { MISSCARE } \\
\text { Survey }\end{array}$ & $\begin{array}{l}\text { A small sample of } \\
\text { staff nurses } \\
(\mathrm{n}=188) \text { working } \\
\text { in general wards } \\
\text { and ICU was } \\
\text { obtained from one } \\
\text { university tertiary } \\
\text { hospital. }\end{array}$ & $\begin{array}{l}\text { Both } \\
\text { correlational } \\
\text { analyses and } \\
\text { multiple } \\
\text { regression } \\
\text { analyses } \\
\text { were } \\
\text { employed. }\end{array}$ & $\begin{array}{l}\text { Nurse manager } \\
\text { ability, leadership, } \\
\text { and support of } \\
\text { nurses }(\beta=-0.26 \text {; } \\
p=0.004) \text {, staffing } \\
\text { and resource } \\
\text { adequacy } \\
(\beta=-0.31 ; \\
p=0.001), \\
(\beta=-0.19 \text {, } \\
p=0.041) \text {, were } \\
\text { found as the } \\
\text { influential factors. } \\
\text { Some dimensions } \\
\text { of the PES-NWI } \\
\text { were not confirmed } \\
\text { to be significant } \\
\text { predictors of UNC. }\end{array}$ & $\begin{array}{l}\text { The cross- } \\
\text { sectional } \\
\text { design allows } \\
\text { for no causal } \\
\text { inferences. } \\
\text { Validated } \\
\text { measurements } \\
\text { were used. } \\
\text { The results } \\
\text { have limited } \\
\text { generalizability. }\end{array}$ \\
\hline $\begin{array}{l}\text { Lake et al. } \\
\text { (2017), } \\
\text { California, } \\
\text { Florida, New } \\
\text { Jersey and } \\
\text { Pennsylvania }\end{array}$ & PES-NWI & $\begin{array}{l}\text { The questions } \\
\text { about } 12 \\
\text { nursing care } \\
\text { "activities } \\
\text { which were } \\
\text { necessary but } \\
\text { left undone } \\
\text { because the } \\
\text { nurses lacked } \\
\text { the time to } \\
\text { complete } \\
\text { them" on the } \\
\text { most recent } \\
\text { shift worked } \\
\text { were } \\
\text { developed. }\end{array}$ & $\begin{array}{l}\text { The sample consists } \\
\text { of } 2,187 \text { paediatric } \\
\text { nurses from } 223 \\
\text { hospitals working } \\
\text { on ICU and acute } \\
\text { paediatrics. }\end{array}$ & $\begin{array}{l}\text { Logistic and } \\
\text { linear } \\
\text { regression } \\
\text { models were } \\
\text { used. }\end{array}$ & $\begin{array}{l}\text { MC was more } \\
\text { common in poor } \\
\text { versus better work } \\
\text { environments }(1.9 \\
\text { vs } 1.2 ; \mathrm{p}<0.01) \text {. } \\
\text { For } 9 \text { of } 12 \text { nursing } \\
\text { activities, the } \\
\text { prevalence of MC } \\
\text { was significantly } \\
\text { higher in poor } \\
\text { environments } \\
\text { (p }<0.05) \text { The } \\
\text { largest work } \\
\text { environment effects } \\
\text { were observed for } \\
\text { the timely } \\
\text { administration of } \\
\text { medications and } \\
\text { patient surveillance } \\
(\text { OR }=0.47 \text { ). } \\
\text { The odds that a } \\
\text { nurse missed care } \\
\text { were } 40 \% \text { lower in } \\
\text { better work } \\
\text { environments and } \\
\text { increased by } 70 \% \\
\text { for each additional } \\
\text { patient. }\end{array}$ & $\begin{array}{l}\text { The cross- } \\
\text { sectional } \\
\text { design allows } \\
\text { for no causal } \\
\text { inferences. } \\
\text { Validated } \\
\text { measurements } \\
\text { were used only } \\
\text { for the } \\
\text { measurement } \\
\text { of the NWE. } \\
\text { The results } \\
\text { have limited } \\
\text { generalizability } \\
\text { (the data from } \\
2006 \text { to } 2008 \\
\text { were collected). }\end{array}$ \\
\hline
\end{tabular}


Table 1 Description of studies used in the scoping review (Part 5)

\begin{tabular}{|c|c|c|c|c|c|c|}
\hline Study & $\begin{array}{l}\text { NWE } \\
\text { measurements }\end{array}$ & $\begin{array}{l}\text { UNC } \\
\text { measurements }\end{array}$ & $\begin{array}{l}\text { Participants, } \\
\text { hospitals and } \\
\text { units }\end{array}$ & $\begin{array}{l}\text { Data } \\
\text { analysis }\end{array}$ & Main results & $\begin{array}{l}\text { Validity / } \\
\text { reliability }\end{array}$ \\
\hline $\begin{array}{l}\text { Lake et al. } \\
\text { (2018), } \\
\text { California, } \\
\text { Pennsylvania, } \\
\text { Florida, New } \\
\text { Jersey }\end{array}$ & PES-NWI & $\begin{array}{l}\text { The questions } \\
\text { about } 12 \\
\text { nursing care } \\
\text { "activities } \\
\text { which were } \\
\text { necessary but } \\
\text { left undone } \\
\text { because the } \\
\text { nurses lacked } \\
\text { the time to } \\
\text { complete } \\
\text { them" on the } \\
\text { most recent } \\
\text { shift worked } \\
\text { was } \\
\text { developed. }\end{array}$ & $\begin{array}{l}\text { A retrospective, } \\
\text { secondary analysis } \\
\text { of } 1,037 \text { staff } \\
\text { licensed nurses was } \\
\text { performed on } 134 \\
\text { NICUs in four large } \\
\text { U.S. states ( } 39 \% \\
\text { response rate). The } \\
\text { nurses were } \\
\text { classified into three } \\
\text { groups based on } \\
\text { their percent of } \\
\text { infants of black } \\
\text { race. }\end{array}$ & $\begin{array}{l}\text { Regression } \\
\text { models } \\
\text { were used. }\end{array}$ & $\begin{array}{l}\text { A better practice } \\
\text { environment (i.e., a } \\
\text { one unit increase in } \\
\text { the PES-NWI } \\
\text { composite score) } \\
\text { decreased the odds of } \\
\text { MC by more than } \\
\text { half }(\mathrm{OR}=0.32 ; 95 \% \\
\mathrm{CI}=0.20-0.53 ; \\
\mathrm{p}=0.00) .\end{array}$ & $\begin{array}{l}\text { The cross- } \\
\text { sectional } \\
\text { design allows } \\
\text { for no causal } \\
\text { inferences. } \\
\text { Validated } \\
\text { measurements } \\
\text { were used. } \\
\text { The results } \\
\text { have limited } \\
\text { generalizability } \\
\text { (the data were } \\
\text { from } 2006 \text {, } \\
\text { some data for } \\
\text { PES-NWI } \\
\text { were missing } \\
\text { in up to } 2.8 \% \\
\text { of cases). }\end{array}$ \\
\hline $\begin{array}{l}\text { Lake et al. } \\
(2020 a), \\
\text { California, } \\
\text { Florida, New } \\
\text { Jersey and } \\
\text { Pennsylvania }\end{array}$ & PES-NWI & $\begin{array}{l}\text { Nurses } \\
\text { answered } \\
\text { whether } 12 \\
\text { nursing care } \\
\text { activities were } \\
\text { necessary but } \\
\text { left undone } \\
\text { because they } \\
\text { lacked the time } \\
\text { to complete } \\
\text { them' during } \\
\text { the most recent } \\
\text { shift they } \\
\text { worked (two } \\
\text { of them were } \\
\text { then omitted } \\
\text { due to lack of } \\
\text { clinical } \\
\text { relevance in } \\
\text { the unique } \\
\text { L\&D setting). }\end{array}$ & $\begin{array}{l}\text { Data from } 1,313 \\
\text { L\&D registered } \\
\text { nurses in } 247 \\
\text { hospitals from a } \\
\text { four-state nurse } \\
\text { survey conducted } \\
\text { between } 2005- \\
2008 \text { in four large } \\
\text { U.S. states ( } 39 \\
\text { percent response } \\
\text { rate) were used. }\end{array}$ & $\begin{array}{l}\text { Regression } \\
\text { models and } \\
\text { ANOVA } \\
\text { were } \\
\text { employed. }\end{array}$ & $\begin{array}{l}\text { Nurses on average } \\
\text { missed } 1.25 \text { of } 10 \\
\text { activities. The MC } \\
\text { frequency was } 0.89 \\
\text { lower in better work } \\
\text { environments } \\
\text { compared with poor } \\
\text { work environments. } \\
\text { In better as compared } \\
\text { to poor NWEs, the } \\
\text { odds } \\
\text { (OR = 0.38) and } \\
\text { frequency } \\
\text { (CI = -0.89) of MC } \\
\text { was significantly } \\
\text { lower. The mean OR } \\
\text { across significant MC } \\
\text { activities was } 0.39 \\
\text { for good NWEs. Pain } \\
\text { management was the } \\
\text { MC activity on which } \\
\text { the NWE had the } \\
\text { largest effect } \\
\text { (OR = 0.30). Other } \\
\text { significant activities } \\
\text { identified included } \\
\text { care planning, } \\
\text { comforting / talking } \\
\text { with patients, } \\
\text { teaching / counselling } \\
\text { patients and families, } \\
\text { care documentation, } \\
\text { patient surveillance } \\
\text { and care } \\
\text { coordination. }\end{array}$ & $\begin{array}{l}\text { The cross- } \\
\text { sectional } \\
\text { design allows } \\
\text { for no causal } \\
\text { inferences. } \\
\text { Validated } \\
\text { measurements } \\
\text { were used only } \\
\text { for the } \\
\text { measurement } \\
\text { of the NWE. } \\
\text { The results } \\
\text { have limited } \\
\text { generalizability } \\
\text { (the data were } \\
\text { from } \\
\text { 2005-2008). }\end{array}$ \\
\hline
\end{tabular}


Table 1 Description of studies used in the scoping review (Part 6)

\begin{tabular}{|c|c|c|c|c|c|c|}
\hline Study & $\begin{array}{l}\text { NWE } \\
\text { measurements }\end{array}$ & $\begin{array}{l}\text { UNC } \\
\text { measurements }\end{array}$ & $\begin{array}{l}\text { Participants, } \\
\text { hospitals and } \\
\text { units }\end{array}$ & $\begin{array}{l}\text { Data } \\
\text { analysis }\end{array}$ & Main results & $\begin{array}{l}\text { Validity / } \\
\text { reliability }\end{array}$ \\
\hline $\begin{array}{l}\text { Lake et al. } \\
(2020 b), \\
\text { California, } \\
\text { Florida, New } \\
\text { Jersey and } \\
\text { Pennsylvania }\end{array}$ & PES-NWI & $\begin{array}{l}\text { Measured by } \\
\text { using the } \\
\text { survey question } \\
\text { "On the most } \\
\text { recent } \\
\text { shift / day you } \\
\text { worked, which } \\
\text { of the } \\
\text { following } \\
\text { activities were } \\
\text { necessary but } \\
\text { left undone } \\
\text { because of time } \\
\text { constraints?" }\end{array}$ & $\begin{array}{l}\text { The data used } \\
\text { were from } \\
23,650 \text { nurses } \\
\text { surveyed in } 2006 \\
\text { and } 14,935 \\
\text { surveyed in } 2016 \\
\text { in } 458 \text { hospitals } \\
\text { in four large U.S. } \\
\text { states from a } \\
\text { four-state survey. }\end{array}$ & $\begin{array}{l}\text { Regression } \\
\text { models } \\
\text { were used. }\end{array}$ & $\begin{array}{l}\text { In hospitals with } \\
\text { improved work } \\
\text { environments or nurse } \\
\text { staffing, the } \\
\text { prevalence and } \\
\text { frequency of MC } \\
\text { decreased significantly } \\
\text { - in hospitals with } \\
\text { improved (or stable) } \\
\text { work environments: } \\
11 \% \text { (or } 6 \% \text { ) fewer } \\
\text { nurses reported MC } \\
\text { in } 2016 \text { (p }<0.001 \text { ); } \\
0.86 \text { (or } 0.55 \text { ) fewer } \\
\text { care activities were } \\
\text { missed in } 2016 \\
\text { (p }<0.001 \text { ); the } \\
\text { prevalence of MC was } \\
\text { reduced by one SD } \\
\text { and total MC was } \\
\text { reduced by } 1.3 \mathrm{SDs} \text {. }\end{array}$ & $\begin{array}{l}\text { A two-period } \\
\text { panel study } \\
\text { was used. } \\
\text { Validated } \\
\text { measurements } \\
\text { were used only } \\
\text { for the } \\
\text { measurement } \\
\text { of the NWE. } \\
\text { The results } \\
\text { have limited } \\
\text { generalizability } \\
\text { (the scales } \\
\text { were used after } \\
10 \text { years). }\end{array}$ \\
\hline $\begin{array}{l}\text { Papastavrou } \\
\text { et al. (2014), } \\
\text { Cyprus } \\
\text { Republic }\end{array}$ & RPPE & BERNCA & $\begin{array}{l}\text { A sample } \\
\text { consisting of } 318 \\
\text { nurses from ten } \\
\text { medical and } \\
\text { surgical units of } \\
\text { five general } \\
\text { hospitals } \\
\text { participated in } \\
\text { the study. }\end{array}$ & $\begin{array}{l}\text { The } \\
\text { Kendall's } \\
\text { tau } \\
\text { correlation } \\
\text { coefficient } \\
\text { was } \\
\text { employed. }\end{array}$ & $\begin{array}{l}\text { The results showed } \\
\text { that between two } \\
\text { scales there was a } \\
\text { small but significant } \\
\text { correlation ( } \tau=-0.234 \text {; } \\
\mathrm{p}<0.001 \text { ). This } \\
\text { indicated that nurses } \\
\text { who were not satisfied } \\
\text { with their work } \\
\text { environment (low } \\
\text { level on RPPE) also } \\
\text { reported that they } \\
\text { frequently were unable } \\
\text { to perform basic } \\
\text { nursing tasks (high } \\
\text { level on BERNCA). }\end{array}$ & $\begin{array}{l}\text { A descriptive, } \\
\text { correlational, } \\
\text { cross-sectional } \\
\text { design allows } \\
\text { for no causal } \\
\text { inference. } \\
\text { Validated } \\
\text { measurements } \\
\text { were used. } \\
\text { Generalisabilit } \\
\text { y of the results } \\
\text { is limited for } \\
\text { each country. }\end{array}$ \\
\hline $\begin{array}{l}\text { Park et al. } \\
(2018), \text { U.S. } \\
\text { states }\end{array}$ & PES-NWI & $\begin{array}{l}\text { A question } \\
\text { asking RNs } \\
\text { whether or not } \\
\text { they missed } \\
\text { any of the } 16 \\
\text { essential care } \\
\text { activities } \\
\text { because of time } \\
\text { constraints }\end{array}$ & $\begin{array}{l}\text { This study } \\
\text { sample included } \\
1,583 \text { units from } \\
371 \text { U.S. acute } \\
\text { care hospitals, } \\
\text { containing } \\
\text { responses from } \\
31,650 \text { RNs. }\end{array}$ & $\begin{array}{l}\text { Adjusted } \\
\text { multilevel } \\
\text { logistic } \\
\text { regression } \\
\text { analysis } \\
\text { was } \\
\text { performed. }\end{array}$ & $\begin{array}{l}\text { The results showed } \\
\text { that good working } \\
\text { environment units had } \\
63.3 \% \text { lower odds of } \\
\text { having RNs MC } \\
\text { activities than poor } \\
\text { working environment } \\
\text { units (OR = 0.377; } \\
95 \% \text { CI } 0.335-0.425 \text {; } \\
\text { p }<0.001) \text {. Similarly, } \\
\text { moderate working } \\
\text { environment units had } \\
36.7 \% \text { lower odds as } \\
\text { compared to poor } \\
\text { working environment } \\
\text { units }(\mathrm{OR}=0.633 \text {; } \\
0,95 \% \text { CI } 0.568- \\
0.705 ; \mathrm{p}<0.001) \text {. }\end{array}$ & $\begin{array}{l}\text { A descriptive, } \\
\text { correlational } \\
\text { cross-sectional } \\
\text { design allows } \\
\text { for no causal } \\
\text { inference. } \\
\text { Validated } \\
\text { measurements } \\
\text { were used only } \\
\text { for the } \\
\text { measurement } \\
\text { of the NWE. } \\
\text { Generalisabilit } \\
\text { y of the results } \\
\text { is limited for } \\
\text { each country. }\end{array}$ \\
\hline
\end{tabular}


Table 1 Description of studies used in the scoping review (Part 7)

\begin{tabular}{|c|c|c|c|c|c|c|}
\hline Study & $\begin{array}{l}\text { NWE } \\
\text { measurements }\end{array}$ & $\begin{array}{l}\text { UNC } \\
\text { measurements }\end{array}$ & $\begin{array}{l}\text { Participants, } \\
\text { hospitals and } \\
\text { units }\end{array}$ & Data analysis & Main results & $\begin{array}{l}\text { Validity / } \\
\text { reliability }\end{array}$ \\
\hline $\begin{array}{l}\text { Pereira } \\
\text { Lima Silva } \\
\text { et al. } \\
(2020) \text {, } \\
\text { Brasil }\end{array}$ & PES-NWI & $\begin{array}{l}\text { The } \\
\text { MISSCARE- } \\
\text { BRASIL } \\
\text { instrument }\end{array}$ & $\begin{array}{l}\text { A small sample } \\
\text { of } \\
\text { professionals } \\
(\mathrm{n}=29) \\
\text { working in } \\
\text { three ICUs was } \\
\text { obtained. }\end{array}$ & $\begin{array}{l}\text { Both } \\
\text { descriptive } \\
\text { and } \\
\text { comparative } \\
\text { analyses were } \\
\text { used. }\end{array}$ & $\begin{array}{l}\text { ICU } 1 \text { presented } \\
\text { a worse mean of } \\
\text { evaluation in the PES } \\
\text { subscale "Adequacy } \\
\text { of the staff and } \\
\text { resources". This ICU } \\
\text { also exhibited the } \\
\text { highest percentage } \\
\text { of omission of care. } \\
\text { In ICU 2, which is } \\
\text { considered to be } \\
\text { a "favourable" } \\
\text { working environment, } \\
\text { there was retained the } \\
\text { lowest rate of } \\
\text { omission of care. } \\
\text { It was proved, that } \\
\text { the professional } \\
\text { practice environment, } \\
\text { as well as the } \\
\text { workload, may be } \\
\text { predictive factors for } \\
\text { the omission } \\
\text { of assistance. }\end{array}$ & $\begin{array}{l}\text { A descriptive, } \\
\text { quantitative and } \\
\text { cross-sectional } \\
\text { design allows for } \\
\text { no causal } \\
\text { inference. } \\
\text { Validated } \\
\text { measurements } \\
\text { were used. } \\
\text { Generalisability } \\
\text { of the results is } \\
\text { limited for each } \\
\text { country (very } \\
\text { small sample; the } \\
\text { statistical } \\
\text { methods used } \\
\text { cannot be traced). }\end{array}$ \\
\hline $\begin{array}{l}\text { Rochefort } \\
\& \text { Clarke } \\
(2010), \\
\text { Canada }\end{array}$ & NWI-R & NEWRI & $\begin{array}{l}\text { The sample } \\
\text { consisted of } \\
339 \text { nurses } \\
(61.3 \%) \\
\text { working in } \\
\text { nine NICUs) } \\
\text { from the } \\
\text { province of } \\
\text { Quebec. }\end{array}$ & $\begin{array}{l}\text { Linear } \\
\text { regression } \\
\text { models were } \\
\text { fitted. }\end{array}$ & $\begin{array}{l}\text { A favourable work } \\
\text { environment was } \\
\text { related to lower levels } \\
\text { of reported nursing } \\
\text { care rationing. The } \\
\text { rationing of parental } \\
\text { teaching, support, } \\
\text { infant comfort care, } \\
\text { discharge planning } \\
\text { and care coordination } \\
\text { was approximately } \\
11 \% \text { lower when } \\
\text { nurse staffing and } \\
\text { resource adequacy } \\
\text { were rated. Similarly, } \\
\text { reductions } \\
\text { of respectively } 5.7 \% \\
\text { and } 7.7 \% \text { in the } \\
\text { rationing of life } \\
\text { support, } \\
\text { technologically } \\
\text { oriented nursing care } \\
\text { and patient } \\
\text { surveillance would be } \\
\text { expected between the } \\
\text { best and worst ratings } \\
\text { of nurse staffing and } \\
\text { resource adequacy. }\end{array}$ & $\begin{array}{l}\text { A cross-sectional } \\
\text { correlational } \\
\text { survey design } \\
\text { allows for no } \\
\text { causal inference. } \\
\text { Validated } \\
\text { measurements } \\
\text { were used. } \\
\text { Generalisability } \\
\text { of the results is } \\
\text { limited within the } \\
\text { country in } \\
\text { question. }\end{array}$ \\
\hline
\end{tabular}


Table 1 Description of studies used in the scoping review (Part 8)

\begin{tabular}{|c|c|c|c|c|c|c|}
\hline Study & $\begin{array}{l}\text { NWE } \\
\text { measurements }\end{array}$ & $\begin{array}{l}\mathrm{UNC} \\
\text { measurements }\end{array}$ & $\begin{array}{l}\text { Participants, } \\
\text { hospitals } \\
\text { and units }\end{array}$ & $\begin{array}{l}\text { Data } \\
\text { analysis }\end{array}$ & Main results & $\begin{array}{l}\text { Validity / } \\
\text { reliability }\end{array}$ \\
\hline $\begin{array}{l}\text { Schubert et } \\
\text { al. (2013), } \\
\text { German, } \\
\text { French, } \\
\text { Switzerland }\end{array}$ & PES - NWI-R & BERNCA & $\begin{array}{l}\text { Data from } \\
1,633 \\
\text { registered } \\
\text { nurses } \\
\text { working in } \\
\text { randomly } \\
\text { selected } \\
\text { medical, } \\
\text { surgical or } \\
\text { medical- } \\
\text { surgical units } \\
\text { of acute care } \\
\text { hospitals } \\
\text { from the } \\
\text { German, } \\
\text { French and } \\
\text { Italian } \\
\text { speaking } \\
\text { regions of } \\
\text { Switzerland } \\
\text { were } \\
\text { collected. }\end{array}$ & $\begin{array}{l}\text { Nurse survey } \\
\text { data were } \\
\text { used from the } \\
\text { RN4CAST- } \\
\text { study (2009- } \\
\text { 2010). } \\
\text { Multilevel } \\
\text { regression } \\
\text { analyses } \\
\text { were } \\
\text { developed. }\end{array}$ & $\begin{array}{l}\text { It was proved that the } \\
\text { unit level staff resource } \\
\text { adequacy, a dimension } \\
\text { of the nurse practice } \\
\text { environment, was } \\
\text { significantly associated } \\
\text { with rationing } \\
(\beta=0.181 ; \mathrm{p}<0.001 \text { ). } \\
\text { Moreover, the two } \\
\text { studied nurse practice } \\
\text { environment } \\
\text { dimensions - "nurse } \\
\text { manager ability", and } \\
\text { "collegial nurses } \& \\
\text { physicians" were not, as } \\
\text { proposed, significantly } \\
\text { associated with } \\
\text { rationing, either in the } \\
\text { separate } \\
\text { (p-value = 0.062-0.866) } \\
\text { or in the adjusted model } \\
\text { (p-value = 0.186-0.905). }\end{array}$ & $\begin{array}{l}\text { The cross- } \\
\text { sectional } \\
\text { design allows } \\
\text { for no causal } \\
\text { inferences. } \\
\text { Validated } \\
\text { measurements } \\
\text { were used. }\end{array}$ \\
\hline $\begin{array}{l}\text { Smith et al. } \\
(2020 a) \text {, U.S. } \\
\text { states }\end{array}$ & $\begin{array}{l}\text { JES } \\
\text { PES-NWI } \\
\text { The impact of } \\
\text { PES-NWI and } \\
\text { TU was testing } \\
\text { with JES. } \\
\text { There wasn't } \\
\text { testing } \\
\text { association } \\
\text { between PES- } \\
\text { NWI and TU. }\end{array}$ & $\begin{array}{l}\text { Measured by } \\
\text { the following } \\
\text { question that } \\
\text { included } 17 \\
\text { care activities } \\
\text { specific to } \\
\text { NICU: "On the } \\
\text { most recent } \\
\text { shift you } \\
\text { worked, which } \\
\text { of the } \\
\text { following } \\
\text { nursing } \\
\text { activities were } \\
\text { necessary but } \\
\text { left undone due } \\
\text { to time } \\
\text { constraints?" }\end{array}$ & $\begin{array}{l}\text { There were } \\
5,824 \text { staff, } \\
\text { RNs, } 303 \\
\text { NICUs and } \\
280 \text { hospitals } \\
\text { from U.S. } \\
\text { states } \\
\text { included. }\end{array}$ & $\begin{array}{l}\text { Secondary } \\
\text { data from the } \\
2016 \\
\text { National } \\
\text { Database of } \\
\text { Nursing } \\
\text { Quality } \\
\text { Indicators } \\
\text { Registered } \\
\text { Nurse Survey } \\
\text { were used. } \\
\text { Both linear } \\
\text { and logistic } \\
\text { regressions } \\
\text { were } \\
\text { calculated. }\end{array}$ & $\begin{array}{l}\text { The missed nursing care } \\
\text { was associated with } \\
\text { a } 0.35 \text { SD decrease in } \\
\text { the job enjoyment scale. } \\
\text { A one SD increase in the } \\
\text { work environment score } \\
\text { was associated with } \\
\text { a } 0.36 \text { SD significant } \\
\text { increase in job } \\
\text { enjoyment. }\end{array}$ & $\begin{array}{l}\text { The cross- } \\
\text { sectional } \\
\text { design allows } \\
\text { for no causal } \\
\text { inferences. } \\
\text { Validated } \\
\text { measurements } \\
\text { were used only } \\
\text { for the } \\
\text { measurement } \\
\text { of the NWE. } \\
\text { The results } \\
\text { have limited } \\
\text { generalizability } \\
\text {. }\end{array}$ \\
\hline $\begin{array}{l}\text { Smith et al. } \\
(2020 b) \text {, } \\
\text { Australia }\end{array}$ & NWI-R-A & $\begin{array}{l}\text { Assessed by } \\
\text { asking nurses } \\
\text { to identify } \\
\text { from a list of } \\
13 \text { activities } \\
\text { which items } \\
\text { were } \\
\text { considered to } \\
\text { be "Necessary } \\
\text { but left undone } \\
\text { because you } \\
\text { lacked time to } \\
\text { complete } \\
\text { them?" during } \\
\text { their last } \\
\text { nursing shift. }\end{array}$ & $\begin{array}{l}\text { A total of } 383 \\
\text { nurses from } \\
\text { small } \\
\text { Australian } \\
\text { rural } \\
\text { hospitals } \\
\text { completed } \\
\text { the survey. }\end{array}$ & $\begin{array}{l}\text { Pearson's r } \\
\text { and } \\
\text { Independent- } \\
\text { sample t-tests } \\
\text { were used. }\end{array}$ & $\begin{array}{l}\text { There were moderately } \\
\text { significant negative } \\
\text { correlations between } \\
\text { care left undone and the } \\
\text { NWI-R: a total mean } \\
(\mathrm{r}=-0.36 ; \mathrm{p}<0.01) \text {. } \\
\text { Nurses who reported } \\
\text { leaving care left undone } \\
\text { had statistically } \\
\text { significant lower } \\
\text { perceptions of the } \\
\text { nursing practice } \\
\text { environment ( }<<0.001) \text {. } \\
\text { The effect sizes ranged } \\
\text { from } 0.25 \text { to } 0.51 \\
\text { reflecting moderate to } \\
\text { medium differences } \\
\text { in nursing practice } \\
\text { environments. }\end{array}$ & $\begin{array}{l}\text { The } \\
\text { descriptive, } \\
\text { cross-sectional } \\
\text { design allows } \\
\text { for no causal } \\
\text { inferences. } \\
\text { Validated } \\
\text { measurements } \\
\text { were used only } \\
\text { for the } \\
\text { measurement } \\
\text { of the NWI-R: } \\
\text { A. } \\
\text { The results } \\
\text { have limited } \\
\text { generalizability } \\
\text { - }\end{array}$ \\
\hline
\end{tabular}


Table 1 Description of studies used in the scoping review (Part 9)

\begin{tabular}{|c|c|c|c|c|c|c|}
\hline Study & $\begin{array}{l}\text { NWE } \\
\text { measurements }\end{array}$ & $\begin{array}{l}\text { UNC } \\
\text { measurements }\end{array}$ & $\begin{array}{l}\text { Participants, } \\
\text { hospitals and } \\
\text { units }\end{array}$ & $\begin{array}{l}\text { Data } \\
\text { analysis }\end{array}$ & Main results & $\begin{array}{l}\text { Validity / } \\
\text { reliability }\end{array}$ \\
\hline $\begin{array}{l}\text { Zeleníková } \\
\text { et al. } \\
(2020) \text {, } \\
\text { Czech } \\
\text { Republic }\end{array}$ & RPPE & $\begin{array}{l}\text { The } \\
\text { MISSCARE } \\
\text { survey }\end{array}$ & $\begin{array}{l}\text { The sample } \\
\text { included } 513 \\
\text { general and } \\
\text { practical nurses } \\
\text { providing direct } \\
\text { care in nine } \\
\text { Czech hospitals. }\end{array}$ & $\begin{array}{l}\text { Spearman's } \\
\text { correlation } \\
\text { coefficient } \\
\text { was used. }\end{array}$ & $\begin{array}{l}\text { The overall score of } \\
\text { missed nursing care } \\
\text { negatively correlated } \\
\text { with the RPPE total } \\
\text { score }(-0.2141) \text {. It } \\
\text { means that a better } \\
\text { professional practice } \\
\text { environment } \\
\text { reflected a lower } \\
\text { level of missed } \\
\text { nursing care. }\end{array}$ & $\begin{array}{l}\text { The descriptive, } \\
\text { cross-sectional } \\
\text { design allows for } \\
\text { no causal } \\
\text { inferences. } \\
\text { Validated } \\
\text { measurements } \\
\text { were used. } \\
\text { The results have } \\
\text { limited } \\
\text { generalizability. }\end{array}$ \\
\hline
\end{tabular}

BERNCA - Basel Extent of Rationing of Nursing Care; $\beta$ - the regression beta coefficient; CI - confidence interval; HCSW - Healthcare Support Workers; ICU - intensive care unit; JES - Job Enjoyment Scale; L\&D - labour and delivery; MC - missed care; MISSCARE survey - Missed Nursing Care Survey; NEWRI - Neonatal Extent of Work Rationing Instrument; NHS - National Health Service; NICU - neonatal intensive care unit; NWE - nursing work environment; NWI-R - Nursing Work Index-Revised; NWI-R-A - Nursing Work Index-Revised: Australian; OR - odds ratio; $p$ - the level of statistical significance; PES-NWI - Practice Environment Scale of the Nursing Work Index; PIRNCA - Perceived Implicit Rationing of Nursing Care Survey; RNs registered nurses; RN4CAST - Nurse Forecasting: Human Resources Planning in Nursing study; RPPE - Revised Professional Practice Environment scale; $r$-correlation coefficient; SD - standard deviation; TU - Task undone tool; UK-United Kingdom; UNC - unfinished nursing care

\section{Discussion}

This scoping review aimed to map the evidence about the impact of the NWE on UNC and to identify which implications are important for further research.

\section{Methodological issues of included studies}

The literature about the NWE and UNC is substantial and continues to expand internationally; however, it is hindered by inconsistencies in the use of terminology, or a wide variety of operationalizations to represent these two constructs (Jones et al., 2019; Norman \& Strømseng Sjetne, 2017). Moreover, the majority instruments for measurement of the NWE and UNC were developed in the United States; thus the dimensions of the NWE or activities included in UNC instruments may not reflect specific work settings in other countries. Most studies in this area focused predominantly on nurses' perceptions of the NWE and UNC. Few studies measured UNC from a patient perspective or with objective measures of objective indicators. Concerning the measurement of UNC, Palese et al. (2019) have emphasized a transition from self-reported UNC measures to objective measures of objective indicators. There was substantial variation in which nursing activities and work environment domains were assessed by the instruments. Interventions related to mobilization / ambulation support, surveillance, and, especially, activities related to emotional and psychological needs (or activities for which the desired time and endeavour are hard to approximate [Mantovan et al., 2020]) were consistently reported among the top five missed activities in both the missed care and in the implicit rationing approach (Bragadóttir et al., 2017; Duffy et al., 2018; Kim et al., 2018; Park et al., 2018; Pereira et al., 2020; Zeleníková et al., 2020).

\section{Relationship between the NWE and UNC}

UNC activities are a mediator between the NWE and patient safety (Liu et al., 2018), or between nursing staffing levels and patient outcomes (Griffiths et al., 2020). A negative relationship was found between nurses' perceptions of patient safety climate and UNC (Ausserhofer et al., 2013; Gurková et al., 2020). Hospital or unit types were found to be a factor contributing significantly to UNC. Statistically significant differences were found between critical care units and mother-baby units (Duffy et al., 2018), and between surgical and medical units (Papastavrou et al., 2014b). Differences in UNC between unit types could be explained by different nurse-patient ratios in various unit types, especially when comparing medical-surgical units and ICUs (Campbell et al., 2020). In qualitative studies, nurses' experiences of UNC were described as "the process of intuitive, situational decision-making and priority setting" (Mantovan et al., 2020). Nurses use several strategies to limit levels of UNC, such as postponing tasks and working overtime, reducing quality, or team strategies (Jones et al., 2015; Mantovan et al., 2020).

Based on the most significant associations found, the NWE was a significant predictor of UNC. The more favorable the new, as perceived by nurses, the less frequently they reported UNC events. Studies using the RPPE showed a low association between UNC and the NWE (Papastavrou et al., 2014b; Zeleníková et al., 2020). On the other hand, some domains of the PES-NWI were found to be significant contributing factors to UNC. Seven studies (Campbell 
et al., 2020; Kim et al., 2018; Park et al., 2018; Rochefort \& Clarke, 2010; Schubert et al., 2013; Smith et al., 2020b; Zeleníková et al., 2020) evaluated associations between specific domains of the PES-NWI and UNC. However, most studies calculated a composite score averaging scores of the dimensions to delineate the overall NWE, which do not provide a basis for specific interventions or supportive strategies for mitigating UNC events. The significant relationship between the NWE and UNC was also confirmed in nursing homes (Zúñiga et al., 2015). Negative associations were found between UNC, five subscales and a composite score of the PES-NWI in two studies (Campbell et al., 2020; Smith et al., 2020).

Improving staffing and resource adequacy was found to be the most significant factor predicting lower prevalence of UNC activities in regression analyses performed in several studies (Kim et al., 2018; Park et al., 2018; Rochefort \& Clarke, 2010; Schubert et al., 2013; Zúñiga et al., 2015). Zhao et al. (2020), in their recent systematic review, have concluded that two domains of the PES-NWI (staffing and resource adequacy; and teamwork) had a great impact on prevalence of UNC. In the South Korean study, two domains of the PES-NWI (nurse manager ability, leadership, and support of nurses; and staffing and resource adequacy) were found to be influential factors of UNC. Park et al. (2018) identified three domains (staffing and resource adequacy; nursephysician relations; and nurse participation in hospital affairs) that were significantly related to UNC.

Recent research syntheses (Cordeiro et al., 2020; Zhao et al., 2020) have emphasized that there is a continuing need to explore the potential differential impact of dimensions of the NWE on UNC events. Almost all included studies had a cross-sectional design that did not allow causal inferences. Refinement of factors in the NWE and staffing are interventions that can mitigate UNC (Lake et al., 2020b). Therefore, longitudinal studies providing causal evidence are needed (Lake et al., 2020b). Stressing the relationship between UNC and organizational changes in longitudinal studies is a potential strategy for improving patient safety (Lake et al., 2020b).

In general, attributes of the NWE have not been systematically examined as important contributing factors of UNC in Central European countries. Previous studies have mainly focused on the prevalence and pattern of UNC, differences in UNC according to a selected hospital or unit variables, and associations between safety climate and UNC (Gurková et al., 2020; Zeleníková et al., 2020). This scoping review is a product of the initial stage of a research project concerned with investigating the association between the NWE and UNC. Based on the results of this scoping review, in the next step of the project we will investigate the relationship between nurses' perceptions of domains of the NWE and UNC in acute care hospitals in the Czech Republic.

\section{Limitation of study}

The search was limited to electronic scientific databases accessible to the authors' institutions, and to publications in English.

\section{Conclusion}

Nurses working in favorable conditions reported lower prevalence of UNC. Results of studies in which the PES-NWI was used have confirmed that the NWE is a significant predictor of UNC. Seven studies explained the relationship between individual factors or specific domains in the NWE and specific nursing activities left undone. Improved staffing and resource adequacy was found to be the most significant factor predicting lower prevalence of UNC activities. Refinement of the factors in the nursing work environment and in staffing are interventions that can mitigate UNC. Researchers should pay more attention to the relationship between different NWEs and prevalence of unfinished nursing care in hospital settings.

\section{Ethical aspects and conflict of interest}

The authors declare no potential conflicts of interest with respect to the research, authorship and / or publication of this article. The research protocol was approved by the Ethical Committee of the Faculty of Health Sciences of Palacký University Olomouc.

\section{Funding}

This article is based upon work from Grant IGA Unfinished nursing care and practice working environment (IGA_FZV_2020_001).

\section{Author contributions}

Concept and design (EG), analysis and data interpretation (EG, DB, ZM), preparation of the manuscript (EG, DB), critical revision of manuscript (EG, DB, ZM), final revision (EG, DB, ZM).

\section{References}

Aiken, L. H., Sloane, D. M., Ball, J., Bruyneel, L., Rafferty, A. M., \& Griffiths, P. (2018). Patient satisfaction with hospital care and nurses in England: an observational study. $B M J$ Open, 8, e019189. https://doi.org/10.1136/bmjopen-2017$\underline{019189}$ 
Ausserhofer, D., Schubert, M., Desmedt, M., Blegen, M. A., De Geest, S., \& Schwendimann, R. (2013). The association of patient safety climate and nurse-related organizational factors with selected patient outcomes: a cross-sectional survey. International Journal of Nursing Studies, 50(2), 240252. https://doi.org/10.1016/j.ijnurstu.2012.04.007

Ausserhofer, D., Zander, B., Busse, R., Schubert, M., De Geest, S., Rafferty, A. M., Ball, J., Scott, A., Kinnunen, J., Heinen, M., Strømseng Sjetne, I., Moreno-Casbas, T., Kózka, M., Lindqvist, R., Diomidous, M., Bruyneel, L., Sermeus, W., Aiken, L. H., \& Schwendimann, R. (2014). Prevalence, patterns and predictors of nursing care left undone in European hospitals: results from the multicountry cross-sectional. RN4CAST study. BMJ Quality \& Safety, 23(2), 126-135. https://doi.org/10.1136/bmjqs-2013-002318

Bail, K., \& Grealish, L. (2016). 'Failure to Maintain': a theoretical proposition for a new quality indicator of nurse care rationing for complex older people in hospital. International Journal of Nursing Studies, 63, 146-161. https://doi.org/10.1016/j.ijnurstu.2016.08.001

Ball, J. E., Murrells, T., Rafferty, A. M., Morrow, E., \& Griffiths, P. (2014). 'Care left undone' during nursing shifts: associations with workload and perceived quality of care. BMJ Quality \& Safety, 23, 116-125. https://doi.org/10.1136/bmjqs-2012-001767

Ball, J. E., Lindqvist, R., Griffiths, P., Rafferty, A. M., Murrells, T., \& Tishelman, C. (2016). A cross-sectional study of 'care left undone' on nursing shifts in hospitals. Journal of Advanced Nursing, 72(9), 2086-2097.

https://doi.org/10.1111/jan.12976

Blackman, I., Henderson, J., Willis, E., Hamilton, P., Toffoli, L., Verrall, C., Abery, E., \& Harvey, C. (2015). Factors influencing why nursing care is missed. Journal of Clinical Nursing, 24(1-2), 47-56. https://doi.org/10.1111/jocn.12688

Blackman, I., Papastavrou, E., Palese, A., Vryonides, S., Henderson, J., \& Willis, E. (2018). Predicting variations to missed nursing care: a three-nation comparison. Journal of Nursing Management, 26(1), 33-41.

https://doi.org/10.1111/jonm.12514

Bragadóttir, H., Kalisch, B. J., \& Tryggvadóttir, G. B. (2017). Correlates and predictors of missed nursing care in hospitals. Journal of Clinical Nursing, 26(11-12), 1524-1534. https://doi.org/10.1111/jocn.13449

Brooks Carthon, J. M., Lasater, K. B., Sloane, D. M., \& Kutney-Lee, A. (2015). The quality of hospital work environments and missed nursing care is linked to heart failure readmissions: a cross-sectional study of US hospitals. BMJ Quality \& Safety, 24(4), 255-263.

https://doi.org/10.1136/bmjqs-2014-003346

Campbell, C. M., Prapanjaroensin, A., Anusiewicz, C. V., Baernholdt, M., Jones, T., \& Patrician, P. A. (2020). Variables associated with missed nursing care in Alabama: a cross-sectional analysis. Journal of Nursing Management, 28(8), 2174-2184. https://doi.org/10.1111/jonm.12979

Cordeiro, R., Serra, R. D., Rodriguez, M. J. P., \& Calha, A. (2020). Good practices to reduce unfinished nursing care: an integrative review. Journal of Nursing Management, 28(8), 1798-1804.

https://doi.org/10.1111/jonm.12972

Duffy, J. R., Culp, S., \& Padrutt, T. (2018). Description and factors associated with missed nursing care in an acute care community hospital. Journal of Nursing Administration, 48(7-8), 361-367.

https://doi.org/10.1097/NNA.0000000000000630
Griffiths, P., Saville, C., Ball, J., Jones, J., Pattison, N., \& Monks, T. (2020). Nursing workload, nurse staffing methodologies and tools: a systematic scoping review and discussion. International Journal of Nursing Studies, 103, 103487. https://doi.org/10.1016/j.ijnurstu.2019.103487

Gurková, E., Zeleníková, R., Friganovic, A., Uchmanowicz, I., Jarošová, D., Papastavrou, E., \& Žiaková, K. (2020). Hospital safety climate from nurses' perspective in four European countries. International Nursing Review, 67(2), 208-217. https://doi.org/10.1111/inr.12561

Gustafsson, N., Leino-Kilpi, H., Prga, I., Suhonen, R., \& Stolt, M. (2020). Missed care from the patient's perspective - a scoping review. Patient Preference and Adherence, 14, 383400. https://doi.org/10.2147/PPA.S238024

Harvey, C., Thompson, S., Otis, E., \& Willis, E. (2020). Nurses' views on workload, care rationing and work environments. Journal of Nursing Management, 28(4), 912918. https://doi.org/10.1111/jonm.13019

Hessels, A. J., Flynn, L., Cimiotti, J. P., Cadmus, E., \& Gershon, R. R. M. (2015). The impact of the nursing practice environment on missed nursing care. Clinical Nursing Studies, 3(4) , 60-65. https://doi.org/10.5430/cns.v3n4p60

Jones, T. L. (2014). Validation of the Perceived Implicit Rationing of Nursing Care (PIRNCA) instrument. Nursing Forum, 49(2), 77-87. https://doi.org/10.1111/nuf.12076

Jones, T. L., Hamilton, P., \& Murry, N. (2015). Unfinished nursing care, missed care, and implicitly rationed care: state of the science review. International Journal of Nursing Studies, 52(6), 1121-1137.

https://doi.org/10.1016/j.ijnurstu.2015.02.012

Jones, T., Willis, E., Amorim-Lopes, M., Drach-Zahavy, A., \& RANCARE Consortium COST - CA 15208. (2019). Advancing the science of unfinished nursing care: Exploring the benefits of cross-disciplinary knowledge exchange, knowledge integration and transdisciplinarity. Journal of Advanced Nursing, 75(4), 905-917. https://doi.org/10.1111/jan.13948

Kalisch, B. J., \& Williams R. A. (2009). Development and psychometric testing of a tool to measure missed nursing care. Journal of Nursing Administration, 39(5), 211-219. https://doi.org/10.1097/NNA.0b013e3181a23cf5.

Kalisch, B. J., Landstrom, G. L., \& Hinshaw, A. S. (2009). Missed nursing care: a concept analysis. Journal of Advanced Nursing, 65(7), 1509-1517. https://doi.org/10.1111/j.13652648.2009.05027.x

Kalisch, B. J., \& Lee, K. H. (2012). Congruence of perceptions among nursing leaders and staff regarding missed nursing care and teamwork. Journal of Nursing Administration, 42(10), 473-477.

https://doi.org/10.1097/NNA.0b013e31826a1fa4

Kim, K., Yoo, M. S., \& Seo, E. J. (2018). Exploring the influence of nursing work environment and patient safety culture on missed nursing care in Korea. Asian Nursing Research, 12(2), 121-126. https://doi.org/10.1016/j.anr.2018.04.003

Lake, E. T., de Cordova, P. B., Barton, S., Singh, S., Agosto, P. D., Ely, B., Roberts, K. E., \& Aiken, L. H. (2017). Missed nursing care in pediatrics. Hospital Pediatrics, 7(7), 378384. https://doi.org/10.1542/hpeds.2016-0141

Lake, E. T., Staiger, D., Edwards, E. M., Smith, J. G., \& Rogowski, J. A. (2018). Nursing care disparities in neonatal intensive care units. Health Services Research, 53(S1), 3007-3026. https://doi.org/10.1111/1475-6773.12762 
Lake, E. T., French, R., O’Rourke, K., Sanders, J., \& Srinivas, S. K. (2020a). Linking the work environment to missed nursing care in labour and delivery. Journal of Nursing Management, 28(8), 1901-1908. https://doi.org/10.1111/jonm.12856

Lake, E. T., Riman, K. A., \& Sloane, D. M. (2020b). Improved work environments and staffing lead to less missed nursing care: A panel study. Journal of Nursing Management, 28(8), 2157-2165. https://doi.org/10.1111/jonm.12970

Liu, X., Zheng, J., Liu, K., Baggs, J. G., Liu, J., Wu, Y., \& You, L. (2018). Hospital nursing organizational factors, nursing care left undone, and nurse burnout as predictors of patient safety: a structural equation modeling analysis. International Journal of Nursing Studies, 86, 82-89. https://doi.org/10.1016/j.ijnurstu.2018.05.005

Lucero, R. J., Lake, E. T., \& Aiken, L. H. (2009). Variations in nursing care quality across hospitals. Journal of Advanced Nursing, 65(11), 2299-2310. https://doi.org/10.1111/j.13652648.2009.05090.x

Mantovan, F., Muzzana, C., Schubert, P. M., \& Ausserhofer, D. (2020). "It's about how we do it, not if we do it". Nurses' experiences with implicit rationing of nursing care in acute care hospitals: a descriptive qualitative study. International Journal of Nursing Studies, 109, 103688. https://doi.org/10.1016/j.ijnurstu.2020.103688

Norman, R. M., \& Strømseng Sjetne, I. (2017). Measuring nurses' perception of work environment: a scoping review of questionnaires. BMC Nursing, 16, 1-15. https://doi.org/10.1186/s12912-017-0256-9

Palese, A., Bassi, E., Tommasini, C., Vesca, R., Di Falco, A., De Lucia, P., Mulloni, G., Paoletti, F., Rissolo, R., Sist, L., Sanson, G., Guardini, I., Bressan, V., Mesaglio, M., Papastavrou, E., \& Blackman, I. (2019). Developing policies and actions in response to missed nursing care: a consensus process. Journal of Nursing Management, 27(7), 1492-1504. https://doi.org/10.1111/jonm.12835

Papastavrou, E., Andreou, P., \& Efstathiou, G. (2014a). Rationing of nursing care and nurse patient outcomes: a systematic review of quantitative studies. International Journal of Health Planning and Management, 29(1), 3-25. https://doi.org/10.1002/hpm.2160

Papastavrou, E., Andreou, P., Tsangari, H., \& Merkouris, A. (2014b). Linking patient satisfaction with nursing care: the case of care rationing - a correlational study. BMC Nursing, 13, 26. https://doi.org/10.1186/1472-6955-13-26

Park, S. H., Hanchett, M., \& Ma, C. (2018). Practice environment characteristics associated with missed nursing care. Journal of Nursing Scholarship, 50(6), 722-730. https://doi.org/10.1111/jnu.12434

Pereira Lima Silva, R., Gonçalves Menegueti, M., Dias Castilho Siqueira, L., de Araújo, T. R., Auxiliadora-Martins, M., Mantovani Silva Andrade, L., \& Laus, A. M. (2020). Omission of nursing care, professional practice environment, and workload in intensive care units. Journal of Nursing Management, 28(8), 1986-1996. https://doi.org/10.1111/jonm.13005

Peters, M. D. J., Godfrey, C. M., Khalil, H., McInerney, P., Parker, D., \& Soares, C. B. (2015). Guidance for conducting systematic scoping reviews. International Journal of Evidence-Based Healthcare, 13(3), 141-146. https://doi.org/10.1097/XEB.0000000000000050

Rochefort, C. M., \& Clarke, S. P. (2010). Nurses' work environments, care rationing, job outcomes, and quality of care on neonatal units. Journal of Advanced Nursing, 66(10), 2213-2224. 2648.2010.05376.x

Schubert, M., Glass, T. R., Clarke, S. P., Schaffert-Witvliet, B., $\&$ De Geest, S. (2007). Validation of the Basel extent of rationing of nursing care instrument. Nursing Research, 56(6), 416-424.

https://doi.org/10.1097/01.NNR.0000299853.52429.62

Schubert, M., Aussserhofer, D., Desmedt, M., Schwendimann, R., Lesaffre, E., Li, B., \& De Geest, S. (2013). Levels and correlates of implicit rationing of nursing care in Swiss acute care hospitals - a cross sectional study. International Journal of Nursing Studies, 50(2), 230-239.

https://doi.org/10.1016/j.ijnurstu.2012.09.016

Smith, J. G., Rogowski, J. A., \& Lake, E. T. (2020a). Missed care relates to nurse job enjoyment and intention to leave in neonatal intensive care. Journal of Nursing Management, 28(8), 1940-1947. https://doi.org/10.1111/jonm.12943

Smith, S., Lapkin, S., Sim, J., \& Halcomb, E. (2020b). Nursing care left undone, practice environment and perceived quality of care in small rural hospitals. Journal of Nursing Management, 28(8), 2166-2173.

https://doi.org/10.1111/jonm.12975

Tricco, A. C., Lillie, E., Zarin, W., O’Brien, K., Colquhoun, H., Kastner, M., Levac, D., Ng, C., Sharpe, J. P., Wilson, K., Kenny, M., Warren, R., Wilson, C., Stelfox, H. T., \& Straus, S. E. (2016). A scoping review on the conduct and reporting of scoping reviews. BMC Medical Research Methodology, 16, 15. https://doi.org/10.1186/s12874-016-0116-4

Zeleníková, R., Jarošová, D., Plevová, I., \& Janíková, E. (2020). Nurses' perceptions of professional practice environment and its relation to missed nursing care and nurse satisfaction. International Journal of Environmental Research and Public Health, 17(11), 3805. https://doi.org/10.3390/ijerph17113805

Zhao, Y., Ma, D., Wan, Z., Sun, D., Li, H., \& Sun, J. (2020). Associations between work environment and implicit rationing of nursing care: a systematic review. Journal of Nursing Management, 28(8), 1841-1850.

https://doi.org/10.1111/jonm.12895

Zúñiga, F., Ausserhofer, D., Hamers, J. P. H., Engberg, S., Simon, M., \& Schwendimann, R. (2015). The relationship of staffing and work environment with implicit rationing of nursing care in Swiss nursing homes - a cross-sectional study. International Journal of Nursing Studies, 52(9), 14631474. https://doi.org/10.1016/j.ijnurstu.2015.05.005 